\title{
The central nucleus of amygdala is involved in tolerance to the antinociceptive effect of NSAIDs
}

\author{
Merab G. Tsagareli ${ }^{1}$, Nana Tsiklauri ${ }^{1}$, Gulnazi Gurtskaia ${ }^{1}$, Ivliane Nozadze ${ }^{1}$, Elene Abzianidze ${ }^{2}$ \\ ${ }^{1}$ Beritashvili Institute of Physiology, Tbilisi, Georgia; tsagareli@biphysiol.ge \\ ${ }^{2}$ State Medical University of Tbilisi, Tbilisi, Georgia
}

Received 12 October 2009; revised 20 November 2009; accepted 27 November 2009.

\begin{abstract}
Aim: Repeated microinjections of non-opioid analgesics into the midbrain periaqueductal gray matter and rostral ventro-medial medulla induce antinociception with development of tolerance. Antinociception following systemic administration of non-steroidal anti-inflammatory drugs ( $\mathrm{N}$ SAIDs) also exhibit tolerance. Presently our aim was to investigate the development of tolerance to the antinociceptive effects of NSAIDs analgine, ketorolac, and xefocam microinjected into central nucleus of amygdala $(\mathrm{Ce})$ in rats. Methods: Under anesthesia with thiopental a stainless steel guide cannula was stereotaxically implanted unilaterally or bilaterally into the $\mathrm{Ce}$ using stereotaxic atlas coordinates, and anchored to the cranium by dental cement. Five days after surgery, $3 \mu \mathrm{l}$ of these NSAIDs were injected via the injection cannula while the rat was gently restrained. Twenty $\mathrm{min}$ post microinjection, i.e. 10-min before the peak of the drugs' effect is normally reached, animals were tested with tail flick (TF) and hot plate (HP) tests. On the 5th experimental day all animals received a Ce microinjection of morphine. Results: Daily microinjection of NSAIDs into the $\mathrm{Ce}$ uni- or bilaterally, produced antinociception with development of complete tolerance over a 5-day period. Following the treatment period, morphine microinjection into the Ce failed to elicit antinociception, indicating cross-tolerance to the antinociceptive effect of $\mathrm{N}$ SAIDs. In other words, the "non-opioid tolerant" rats showed cross-tolerance to morphine. Conclusions: Our data confirmed the suggestion that NSAIDs interact with endogenous opioid systems, which likely play a key role in the development of tolerance to the antinociceptive effects of NSA IDs.
\end{abstract}

Keywords: Descending Inhibition; Morphine Cross-Tolerance; Nociception

\section{INTRODUCTION}

Microinjection of non-opioid analgesics metamizol, and lysine-acetylsalicylate (LASA) into certain brain areas, including the midbrain periaqueductal gray matter (PAG) and rostral ventro-medial medulla (RVM), produces antinociception with development of some degree of tolerance [1-4]. We have also observed tolerance to the antinociceptive effects of analgine (metamizol), ketorolac, and xefocam administered systemically [5-7]. These studies are consistent with the possibility that endogenous opioidergic mechanisms associated with descending pain modulation may partly mediate the tolerance observed with non-steroidal anti-inflammatory drugs (NSAIDs) [8].

The amygdala receives massive input from the hippocampus and the neocortex and provides a major source of afferents to PAG [9]. Analgesia resulting from microinjection of opioid agonists into the basolateral amygdala is blocked by lidocaine inactivation of, or opioid antagonist injection into, the PAG [10-12]. Cortical afferents to the amygdala largely target its basolateral component. The basolateral amygdala then projects to the central nucleus of amygdala $(\mathrm{Ce})$, which in turn projects densely to the PAG [13]. The Ce also receives nociceptive input, both directly from the spinal cord, and indirectly via a large projection from the dorsal horn to the parabrachial nucleus $[14,15]$. The Ce is an integral component of the endogenous pain-modulatory circuit and is critical for systemic morphine-induced suppression of spinal nociceptive reflexes [16].

The present study reports that microinjection of analgine, ketorolac, and xefocam into the $\mathrm{Ce}$ of rats elicits antinociception with the development of tolerance.

\section{MATERIALS AND METHODS}

The experiments were carried out using male Wistar rats, 200-250g in body weight, bred at the Beritashvili Institute of Physiology. The animals were kept under standard housing conditions $\left(22 \pm 2{ }^{\circ} \mathrm{C}, 65 \%\right.$ humidity, light from 
7:00 a.m. to 8:00 p.m.) and were fed a standard dry diet; water was freely available. Experiments were performed during the light phase of the circle between 10:00 and 14:00 clock. Guidelines of the International Association for the Study of Pain regarding animal experimentation and Guide for the Care and Use of Laboratory Animals (National Academy Press, Washington, DC, 1996) were followed throughout.

Under anesthesia with thiopental $(55 \mathrm{mg} / \mathrm{kg}$, i.p. "Kievmed" Ukraine) a 12-mm stainless steel guide cannula (Plastic One, Inc., USA) was stereotaxically implanted unilaterally (left side) or bilaterally into the Ce amygdala using coordinates from the atlas of Paxinos G. \& Watson C. [17], and anchored to the cranium by dental cement. The guide cannula was plugged with a stainless steel stylet. Thereafter, the rats were handled in 3 daily 15 min periods to become habituated to the experimental environment and test protocol that involved removal of the stylet and insertion of injection cannula $(10 \mathrm{~mm}$ length of PE tubing attached to a $50 \mu \mathrm{l}$ Hamilton syringe; Hamilton, Inc., USA) without drug injection. Five days after surgery, three $\mu l$ of drug was injected via the injection cannula while the rat was gently restrained. Drugs were: Analgine (metamizol sodium, $1.5 \mathrm{mg} / 3 \mu \mathrm{l}$, "Sanitas", Ltd, Lithuania), ketorolac (ketorolac tromethamine, $90 \mu \mathrm{g} / 3 \mu \mathrm{l}$, "Zee Drugs", India), xefocam (lornoxicam, $12 \mu \mathrm{g} / 3 \mu \mathrm{l}$, "Nycomed", GmbH, Austria), or saline $(3 \mu \mathrm{l})$ ("Galichpharm" Ltd. Ukraine). Twenty min post microinjection, i.e. 10min before the peak of the drugs' effect is normally reached, animals were tested with TF or HP. For the TF test, the distal part of the tail was stimulated with a light beam (IITC \#33, IITC Life science, Inc., Woodland Hills, CA, USA) and the latency measured until the tail was reflexively flicked away from the beam. For the HP test, the rat was placed on a $52^{\circ} \mathrm{C}$ hot plate (IITC \#39) and the latency to lick paw or jump was measured. The cut-off time was $20 \mathrm{~s}$ for both TF and HP latencies. Each rat was tested with both tail flick (TF) and hot plate (HP) latencies in the same session. The same procedure was followed to deliver repeated microinjections of each drug (analgine, ketorolac, xefocam) or vehicle (saline) over five consecutive days. On the 5th experimental day all animals received a Ce microinjection of morphine hydrochloride $(3 \mu \mathrm{g} / 2 \mu 1$, "Laboratoires Stella", France) and TF and HP latencies were measured 20 min thereafter. At the conclusion of experiment on the fifth day, the microinjection site was marked with $2 \mu \mathrm{l}$ of a saturated solution of Pontamine Sky Blue (Sigma Chemical Co.,USA), and the animal was sacrificed by ester inhalation. After fixation by immersion in $10 \%$ formalin the brain was sectioned and the microinjection site was identified with the aid of Paxinos \& Watson' stereotaxic atlas [17].

All data are presented as mean \pm S.E.M. Analysis of variance (ANOVA) with post-hoc Tukey-Kramer multiple comparisons were used for statistical evaluations. The statistical software utilized was InStat 3.05 (GraphPad Software, Inc, USA). Statistical significance was acknowledged if $\mathrm{P}<0.05$.

\section{RESULTS}

Only rats with microinjections into Ce were included for data analysis. Histological location of microinjection sites is shown in simplified drawing section from the Paxinos and Watson atlas [17] (Figure 1). These data consisted of 13 rats microinjected with analgine (6 uniand 7 bilaterally), 13 with ketorolac (6 and 7), 12 with xefocam (6 and 6), and 15 with control saline (8 and 7), respectively. Injection sites outside the boundaries of the $\mathrm{Ce}$ (the shaded region in Figure 1) were not included in data analysis. In special control experiments with intentionally microinjections of NSAIDs out of Ce we did not reveal significant changes in TF and HP latencies (data not shown).

On the first test day, unilateral microinjection of each NSAID into the Ce produced antinociception as revealed by significant increases in latency for TF [ANOVA: $\mathrm{F}(3,20)=21.251 ; \mathrm{P}<0.001$ ] and HP [ANOVA: $\mathrm{F}(3,20)=$ 15.872; $\mathrm{P}<0.001]$ compared to saline controls $(\mathrm{P}<0.001$ for all drugs) (Figure 2). However, on successive days, microinjection of each NSAID had a progressively weaker antinociceptive effect such that on the fourth and/or fifth experimental days the TF and HP latencies were not significantly different compared to saline injections. This was similar to the development of tolerance to morphine administration to PAG in similar preparations $[18,19]$, and we therefore refer to it as "non-opioid tolerance". Note, however, that tolerance to the antinociceptive effect of xefocam was slower compared to analgine and ketorolac. On day 5, both experimental and

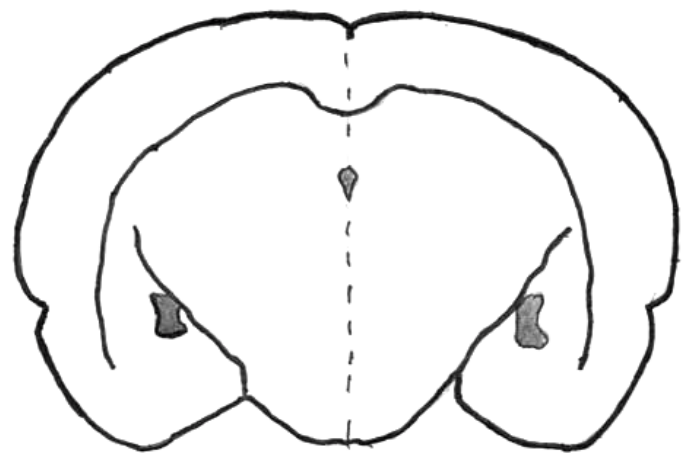

Figure 1. Location of microinjection sites in the Ce. Only NSAIDs and saline injections within the shaded regions were included in data analysis. Coronal sections are taken from the atlas of Paxinos and Watson (1998). The Distances from interaural line $\pm 5.7 \mathrm{~mm}$ and from the bregma $-3.3 \mathrm{~mm}$ respectively. All injections fell within $\pm 0.5 \mathrm{~mm}$ of this coronal plane. 
control groups received a morphine microinjection at the same Ce sites, and only the saline group exhibited antinociception $(\mathrm{P}<0.001)$. The latencies of the non-opioid tolerant rats were not altered by the morphine microinjections, i.e. they showed cross-tolerance to morphine (Figure 2).

Bilateral microinjections into the $\mathrm{Ce}$ also increased latency of TF [ANOVA: $\mathrm{F}(3,20)=8.873 ; \mathrm{P}=0.006$ ] and HP [ANOVA: $\mathrm{F}(3,20)=11.933 ; \mathrm{P}<0.001]$ compared to control rats on the first day for these NSAIDs, in TF $(\mathrm{P}<0.01)$ and in HP $(\mathrm{P}<0.001)$ respectively (Figure 3). Similar to the unilateral drug injections, there was a progressive decline in the antinociceptive effect elicited by bilateral injection of each drug over the 5-day period,
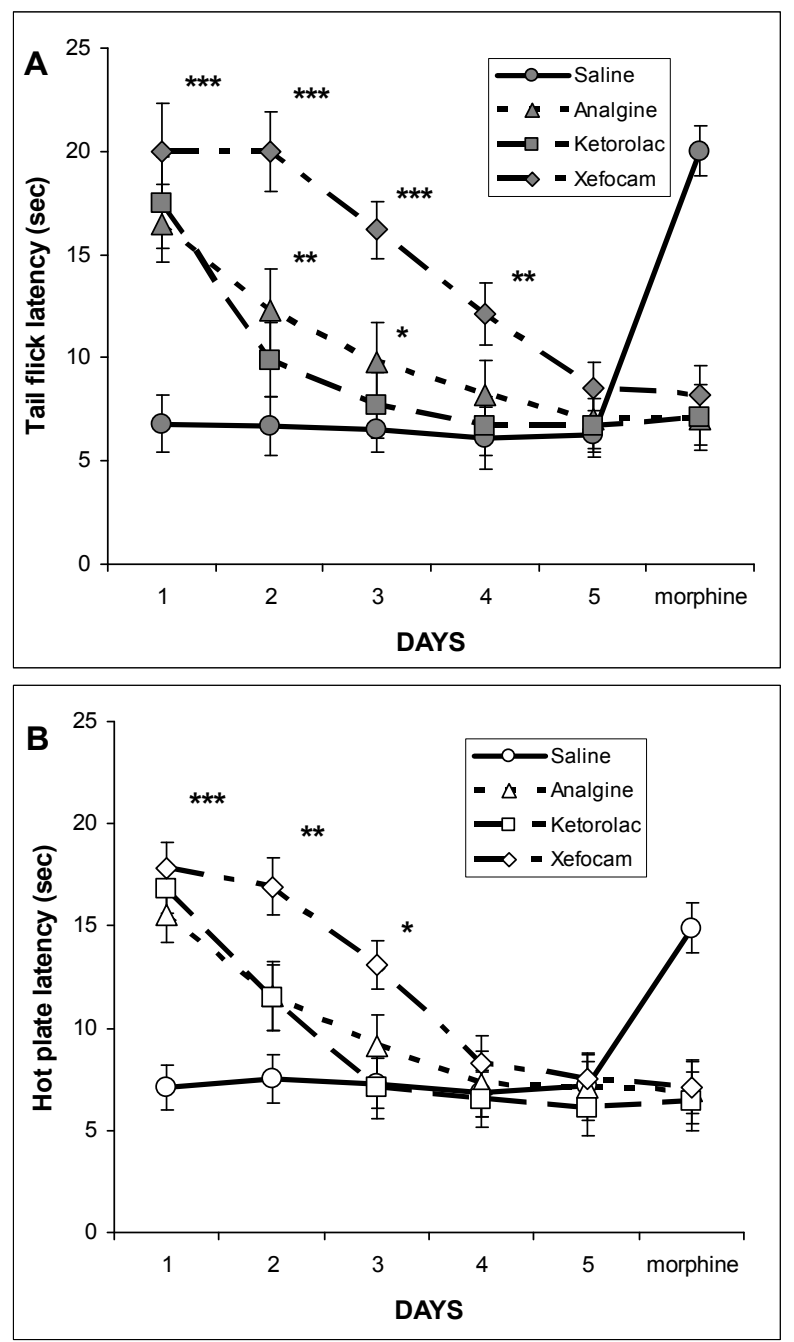

Figure 2. Response latencies in unilateral microinjections. Mean response latencies following unilateral microinjections of each NSAIDs are plotted over the 5-day period, followed by morphine, for TF (A) and HP (B) tests. Cutoff latency (20 sec) was reached in some animals for xefocam and morphine microinjections. Asterisks mark significant difference of latency in comparison to saline control as analyzed by post hoc tests $\left({ }^{*} p<0.05,{ }^{* *} p<0.01,{ }^{* * *} p<0.001\right)$.
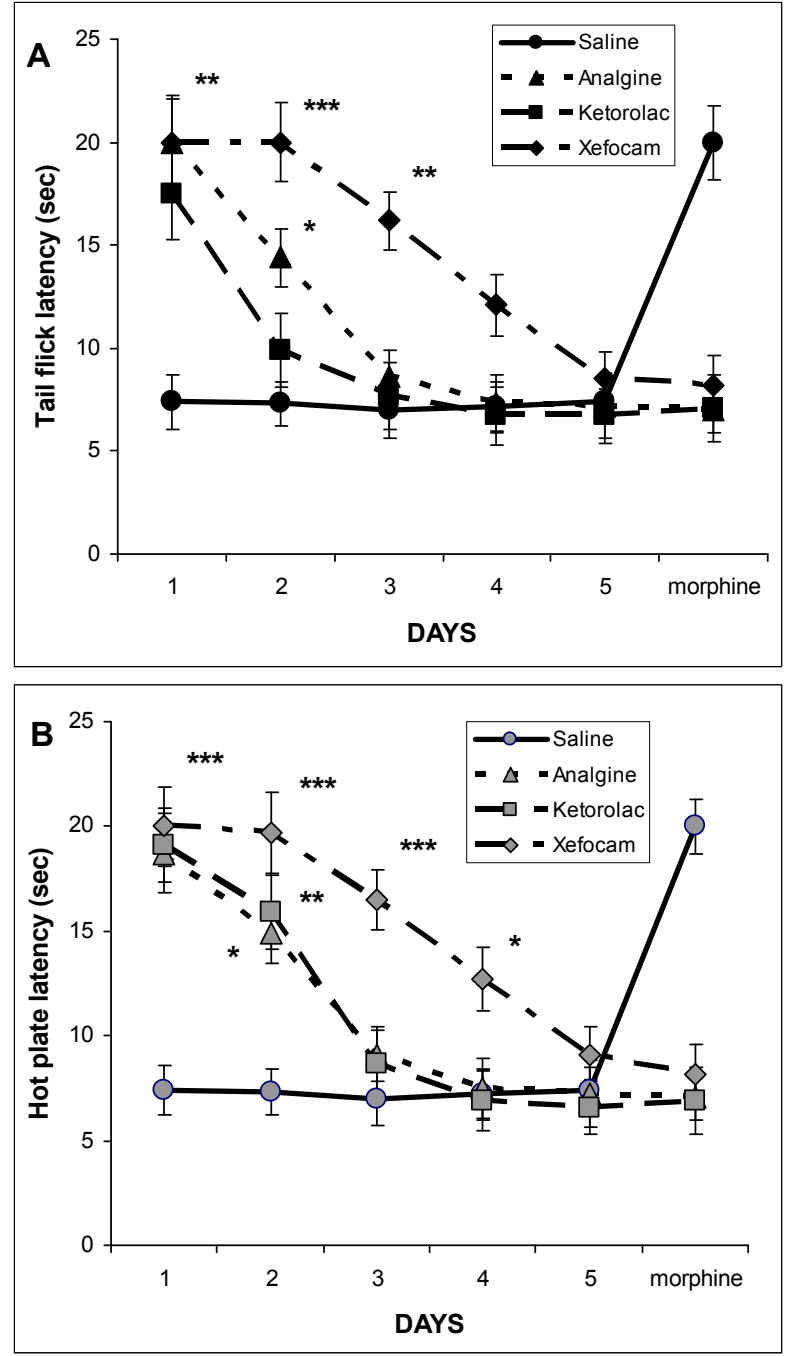

Figure 3. Response latencies in bilateral microinjections. Mean response latencies following bilateral microinjections of each NSAIDs are plotted over the 5-day period, followed by morphine, for TF (A) and HP (B) tests. Cutoff latency (20 sec) was reached in some animals for xefocam and morphine microinjections. Asterisks mark significant difference of latency in comparison to saline control as analyzed by post hoc tests $\left({ }^{*} p<0.05, * * p<0.01, * * * p<0.001\right)$.

such that TF and HP latencies were not significantly different from saline controls after the 4th-5th day (Figure 3). Again, xefocam exhibited a slower time course for development of tolerance. Bilateral microinjection of each NSAIDs also exhibited cross-tolerance to morphine as compared with saline controls $(\mathrm{P}<0.001)$ (Figure 3).

\section{DISCUSSION}

The present study revealed that microinjection of analgine, ketorolac, and xefocam into the Ce induced antinociception in awake rats. This confirmed our previous results with systemic (i.p.) administration of NSAIDs 
[5-7], and results of others using microinjection of the same NSAIDs into the PAG [2-4]. Importantly, repeated microinjections of NSAIDs into the Ce resulted in a progressive decrease in antinociceptive effectiveness (tolerance) similar to that observed with intra-PAG injections [2-4], and reminiscent of the effect of opiates.

A major involvement of opioidergic mechanisms in tolerance to the analgesic effect of NSAIDs was surprising, because traditionally the cellular and molecular actions of opioids were thought to differ from those of nonopioid analgesics. One interesting aspect of NSAIDs administration, namely tolerance, emphasizes their similarity to opioid analgesics. Indeed, microinjection of metamizol $[3,4,20]$, or LASA $[2,20]$ into PAG or into Ce, progressively led to a loss of their antinociceptive effects, i.e. produced tolerance. Furthermore, tolerance to metamizol or LASA was accompanied by cross-tolerance to morphine [2-4] as if opioid analgesics had been repeatedly administered. Interestingly, tolerance to the effect of PAG-microinjected metamizol can, like tolerance to morphine, be reversed by microinjection of proglumide, a cholecystokinin antagonist, at the same PAG site [3]. The latter finding constituted additional evidence that the PAG effects of non-opioid analgesics are similar to those of morphine. Moreover, the data suggested that Ce should be incorporated into current models of endogenous pain control circuitry [21].

It is well known that morphine injection after administration of NSAIDs or in combination, morphine plus NSAIDs usually potentiates their own analgesic effects [8]. We have recently tested each of NSAIDs for crosstolerance to morphine given over a 5-day period in two age groups of rats. There was a significant difference between adult and juvenile rat groups for the degree of morphine analgesia, which was most marked on the first and second experimental days. Furthermore, morphine-tolerant rats exhibited cross-tolerance to analgine, ketorolac, and xefocam for both TF and HP tests, respectively (data not shown).

In conclusion, our data confirmed previous studies indicating that the antinociceptive action of NSAIDs may be closely related to that of endogenous opioids, including the development of tolerance. In addition, the $\mathrm{Ce}$ along with PAG and RVM represents an important component of the endogenous antinociceptive system.

\section{ACKNOWLEDGEMENTS}

The authors would like to thank Professor E. Carstens for helpful comments and English revisions of the manuscript. This research was supported by the grant from Georgian National Science Foundation (GNSF/ST07/6-234).

\section{REFERENCES}

[1] Jones, S.L. (1996) Dipyrone into the nucleus raphe mag- nus inhibits the rat nociceptive tail flick reflex. Eur $J$ Pharmacol, 318, 37-40.

[2] Pernia-Andrade, A.J., Tortorici, V. and Venegas, H. (2002) Induction of opioid tolerance by lysine-acetylsalicylate in rats. Pain, 111, 191-200.

[3] Tortorici, V., Nogueira, L., Salas, R. and Venegas, H. (2003) Involvement of local cholecystokinin in the tolerance induced by morphine microinjections into the periaqueductal gray of rats. Pain, 102, 9-16.

[4] Tortorici, V. and Vanegas, H. (2000) Opioid tolerance induced by metamizol (dipyrone) microinjections into the periaqueductal gray of rats. Eur J Neurosci, 12, 40744080.

[5] Tsagareli, M.G., Tsiklauri, N., Lagidze, T., Gurtskaia, G. and Abzianidze, E. (2005) Tolerance induction by nonopioid analgesics in rats. Proc Georgian Acad Sci Biol Series $A, \mathbf{3 1}, 903-909$.

[6] Tsiklauri, N., Gurtskaia, G. and Tsagareli, M.G. (2006) Is endogenous opioid system involved in non-opioid analgesics tolerance? Georgian Med News, 8(137), 121-125.

[7] Tsiklauri, N. and Tsagareli, M.G. (2006) Non-opioidinduced tolerance in rats. Neurophysiol, 38, 370-373.

[8] Hernandez-Delgadillo, G.P. and Cruz, S.L. (2006) Endogenous opioids are involved in morphine and dipyrone analgesic potentiation in the tail flick test in rats. Eur $J$ Pharmacol, 546, 54-59.

[9] Aggleton, J.P. (Ed) (1996) The amygdala: Neurobiological aspects of emotion, memory, mental dysfunction. Willey-Liss, New York

[10] Bodnar, R.J. (2000) Supraspinal circuitry mediating opioid antinociception: Antagonist and synergy studies in multiple sites. J Biomed Sci, 7, 181-194.

[11] Helmstetter, F.J., Tershner, S.A. and Poore, L.H. (1998) Antinociception following opioid stimulation of the basolateral amygdala is expressed through the periaqueductal grey and rostral ventromedial medulla. Brain Res, 779, 104-118.

[12] Pavlovič, Z., Cooper, M. and Bodnar, R. (1996) Opioid antagonists in the periaqueductal gray inhibit morphine and beta-endorphin analgesia elicited from the amygdala of rats. Brain Res, 741, 13-26.

[13] Rivzi, T.A., Ennis, M. and Behbehani, M.M. (1991) Connections between the central nucleus of the amygdala and the midbrain periaqueductal gray: Topography and reciprocity. J Comp Neurol, 303, 121-131.

[14] Gauriau, C. and Bernard, J.F. (2002) Pain pathways and parabrachial circuits in the rat. Exp Physiol, 87, 251-258.

[15] Gauriau, C. and Bernard, J.F. (2004) A comparative reappraisal of projections from the uperficial laminae of dorsal horn in the rat: forebrain. J Comp Neurol, 468, 24-56.

[16] Manning, B.H. and Mayer, D.J. (1995) The central nucleus of the amygdala contributes to the production of morphine antinociception in the tail-flick test. $J$ Neurosci, 15, 8199-8213.

[17] Paxinos, G. and Watson, C. (1998) The rat brain in stereotaxic coordinates, 4th edition. Academic Press, New York.

[18] Morgan, M.M., Clayton, C.C. and Boyer-Quick, J.S. (2005) Differential susceptibility of the PAG and RVM to tolerance to the antinociceptive effect of morphine in the rat. Pain, 113, 91-98.

[19] Tortorici, V., Nogueira, L., Aponte, Y. and Vanegas, H. 
(2004) Involvement of cholecystokinin in the opioid tolerance induced by dipyrone (metamizol) microinjections into the periaqueductal gray matter of rats. Pain, 112, 113-120.

[20] Vanegas, H. and Tortorici, V. (2002) Opioidergic effects of non-opioid analgesics on the central nervous system. Cell Mol Neurobiol, 22, 655-661.

[21] Price, D.D. (1999) Psychological mechanisms of pain and analgesia. IASP Press, Seattle. 